Check for updates

Cite this: Chem. Sci., 2018, 9, 7413

๑ All publication charges for this article have been paid for by the Royal Society of Chemistry

Received 3rd July 2018

Accepted 1st August 2018

DOI: $10.1039 / \mathrm{c} 8 \mathrm{sc0} 02917 \mathrm{~b}$

rsc.li/chemical-science

\section{Spin-reorientation-induced magnetodielectric coupling effects in two layered perovskite magnets $\dagger$}

\author{
Bo Huang, ${ }^{a}$ Jian-Yu Zhang, (D) ${ }^{a}$ Rui-Kang Huang, ${ }^{a}$ Ming-Kun Chen, ${ }^{a}$ Wei Xue, ${ }^{a}$ \\ Wei-Xiong Zhang, (iD *a Ming-Hua Zeng (iD ${ }^{b}$ and Xiao-Ming Chen ${ }^{a}$
}

Spin-reorientation-induced magnetodielectric coupling effects were discovered in two layered perovskite magnets, $\left[\mathrm{C}_{6} \mathrm{H}_{5} \mathrm{CH}_{2} \mathrm{CH}_{2} \mathrm{NH}_{3}\right]_{2}\left[\mathrm{MCl}_{4}\right]\left(\mathrm{M}=\mathrm{Mn}^{2+}\right.$ and $\left.\mathrm{Cu}^{2+}\right)$, via isothermal magnetodielectric measurements on single-crystal samples. Specifically, peak-like dielectric anomalies and spin-flop transitions appeared simultaneously at around $\pm 34 \mathrm{kOe}$ for the canted antiferromagnet $\left(M=\mathrm{Mn}^{2+}\right)$ at below $44.3 \mathrm{~K}$, while a low-field $(1 \mathrm{kOe})$ controlled magnetodielectric effect was observed in the "soft" ferromagnet $(\mathrm{M}=$ $\left(\mathrm{u}^{2+}\right)$ at below $9.5 \mathrm{~K}$. These isothermal magnetodielectric effects are highly reproducible and synchronous with the field-induced magnetization at different temperatures, well confirming the essential role of spin reorientation on inducing magnetodielectric coupling effects. These findings strongly imply that the layered perovskite magnets are new promising organic-inorganic hybrid systems to host magnetodielectric coupling effects.

\section{Introduction}

The studies of coupling effects between two different physical parameters in molecular materials not only contribute to new perspectives in the fundamental understanding of novel materials, but they also provide extra dimensions for designing multifunctional devices. ${ }^{1}$ Magnetodielectric coupling effects, defined as the dielectric properties controlled by an applied magnetic field, are important for understanding the coupled ferroelectric and magnetic properties in multiferroics, as well as for designing a new generation of magnetodielectric devices. ${ }^{2,3}$

The progress of studying magnetodielectric coupling effects in single-phase materials was very slow for a long time after the first experimental realization in $\mathrm{Cr}_{2} \mathrm{O}_{3}$ in the $1960 \mathrm{~s},{ }^{4}$ until strong magnetodielectric effects were discovered in $\mathrm{TbMnO}_{3}$ in 2003. ${ }^{5}$ Since then, much effort has been devoted to searching for new magnetodielectric materials in different systems of inorganic oxides, and numerous advances have been achieved. ${ }^{6-12}$ For instance, a highly reproducible polarization switching using magnetic fields was observed in the orthorhombic $\operatorname{TbMn}_{2} \mathrm{O}_{5},{ }^{13}$ a gigantic magnetodielectric effect was found in the perovskite-

${ }^{a}$ MOE Key Laboratory of Bioinorganic and Synthetic Chemistry, School of Chemistry, Sun Yat-Sen University, Guangzhou, 510275, P. R. China. E-mail: zhangwx6@mail. sysu.edu.cn

${ }^{b}$ School of Chemistry and Pharmaceutical Sciences, GuangXi Normal University, Guilin 541004, P. R. China

$\dagger$ Electronic supplementary information (ESI) available: Photos of crystals, powder XRD patterns, illustration of hydrogen bonds, and other magnetic properties of PEA-Mn and PEA-Cu. See DOI: 10.1039/c8sc02917b type $\mathrm{YMnO}_{3},{ }^{14}$ and a low-field magnetodielectric effect was found in a Z-type hexaferrite $\mathrm{Sr}_{3} \mathrm{Co}_{2} \mathrm{Fe}_{24} \mathrm{O}_{41} \cdot{ }^{15}$ These outstanding discoveries promoted the research of magnetodielectric coupling effects to the forefront of condensed matter physics and material science.

In parallel to the intensive studies on inorganic oxides, the explorations of magnetodielectric coupling effects in the past decade were extended to organic-inorganic hybrid materials as well, taking advantage of their light weight, mechanical flexibility, environmentally benign synthesis and structural tunability. ${ }^{16,17}$ To date, the major research interest in organicinorganic hybrid materials has been limited to threedimensional metal-formate frameworks, among which more than a few compounds have revealed an interesting coexistence of ferroelectric and antiferromagnetic orders. ${ }^{18-21}$ However, only five compounds, $\left[\mathrm{NH}_{2}\left(\mathrm{CH}_{3}\right)_{2}\right]\left[\mathrm{Fe}^{\mathrm{III}} \mathrm{Fe}_{(1-x)}^{\mathrm{II}} \mathrm{Ni}_{x}^{\mathrm{II}}(\mathrm{HCOO})_{6}\right](x \approx 0.63-$ $0.69),{ }^{22}\left[\left(\mathrm{CH}_{3}\right)_{2} \mathrm{NH}_{2}\right]\left[\mathrm{Mn}(\mathrm{HCOO})_{3}\right],{ }^{23}\left[\left(\mathrm{CH}_{3}\right)_{2} \mathrm{NH}_{2}\right]\left[\mathrm{Fe}(\mathrm{HCOO})_{3}\right],{ }^{24,25}$ $\left(\mathrm{CH}_{3} \mathrm{NH}_{3}\right)\left[\mathrm{Co}(\mathrm{HCOO})_{3}\right],{ }^{26}$ and $\left[\mathrm{NH}_{2}\left(\mathrm{CH}_{3}\right)_{2}\right]\left[\mathrm{Fe}^{\mathrm{III}} \mathrm{Fe}^{\mathrm{II}}(\mathrm{HCOO})_{6}\right],{ }^{27}$ were identified as exhibiting magnetodielectric coupling effects. In this sense, there is still a huge amount of room for progressing the study of magnetodielectric coupling effects in numerous organic-inorganic hybrid materials, ${ }^{28-31}$ and it is urgently needed to investigate more hybrid material systems for exploring stronger magnetodielectric coupling effects.

As a unique family of organic-inorganic hybrids, the twodimensional layered perovskite-type compounds have a general formula of $\mathrm{A}_{2} \mathrm{MX}_{4}$ (the valence ratio of the cation $\mathrm{A}$, metal ion $\mathrm{M}$, and anionic halogen $\mathrm{X}$ components is $1: 2: 1$ ), and structurally consist of infinite inorganic layers of cornersharing $\mathrm{MX}_{6}$ octahedra interleaved by bilayers of organic 
ammonium layers. Comprehensive studies on their magnetic properties have well disclosed that long-range magnetic ordering could be established in the inorganic layers via intraand inter-layer magnetic interactions. Moreover, the magnetic types are strongly dependent on the metal ion $\mathbf{M}$, while the amplitude of the magnetic interactions could be influenced by the halogen ion $\mathrm{X}$ together with the organic cation $\mathrm{A}^{32-34}$ For instance, the layered perovskite-type compounds based on Jahn-Teller active $\mathrm{Cr}^{2+}$ and $\mathrm{Cu}^{2+}$ ions are typically Heisenberg ferromagnets, ${ }^{35,36}$ while the layered perovskites based on $\mathrm{Fe}^{2+}$ and $\mathrm{Mn}^{2+}$ ions are typically antiferromagnets, ${ }^{37-39}$ including some canted antiferromagnets that exhibit a spin-flop transition (vide infra) at low temperature. ${ }^{34}$ It was generally believed that the ferromagnetic ordering could host stronger magnetodielectric interactions than the antiferromagnetic one. Therefore, the ferromagnetic $\mathrm{Cu}^{2+}$-based layered perovskites attracted more attention, and some of them have been assigned as type-I multiferroic materials, ${ }^{33,40,41}$ though none of them have been verified as possessing magnetodielectric coupling effects. In contrast, the spin-flop transition, a field-induced magnetic transition concerning the antiferromagnetic ordering, has rarely been studied for its potentiality to induce magnetodielectric effects in organic-inorganic hybrid materials. ${ }^{26}$

In our previous studies on multiferroic materials based on a ferromagnetic layered perovskite, $\left(\mathrm{C}_{6} \mathrm{H}_{5}\left(\mathrm{CH}_{2}\right)_{4} \mathrm{NH}_{3}\right)_{2}\left[\mathrm{CuCl}_{4}\right]$, we found a coexistence of magnetic and dielectric bistabilities in the vicinity of both ferroelectric and ferroelastic structural phase transitions, arising from the buckling of the octahedra together with the freezing of the organic cations. ${ }^{41}$ Such coexistence of both magnetic and dielectric bistabilities strongly implies that the unique hydrogen-bonding interaction between the inorganic layers and the organic cations may mediate the magnetic and dielectric properties to achieve a coupling effect.

To explore the magnetodielectric coupling effects in layered perovskites, we focused on two known layered perovskite magnets, a canted antiferromagnet $\left(\mathrm{C}_{6} \mathrm{H}_{5} \mathrm{CH}_{2} \mathrm{CH}_{2} \mathrm{NH}_{3}\right)_{2}\left[\mathrm{MnCl}_{4}\right]$ (PEA-Mn) and a ferromagnet $\left(\mathrm{C}_{6} \mathrm{H}_{5} \mathrm{CH}_{2} \mathrm{CH}_{2} \mathrm{NH}_{3}\right)_{2}\left[\mathrm{CuCl}_{4}\right]$ (PEA$\mathrm{Cu}$. PEA-Mn and PEA-Cu are isomorphous, and both of them crystallize in the centrosymmetric space group $\mathrm{Pbca}$ at room temperature. $^{33,34}$ Their structures consist of inorganic layers constructed from corner-sharing $\mathrm{MCl}_{6}$ octahedra $\left(\mathrm{M}=\mathrm{Mn}^{2+}\right.$ and $\mathrm{Cu}^{2+}$ ). The intralayer cavities between the octahedra are occupied by two $-\mathrm{NH}_{3}$ groups of two organic $\mathrm{C}_{6} \mathrm{H}_{5} \mathrm{CH}_{2} \mathrm{CH}_{2} \mathrm{NH}_{3}{ }^{+}$ cations from the up and down sides, separately (Fig. 1a). Notably, such a hybrid structure is stabilized by the $\mathrm{N}-\mathrm{H} \cdots \mathrm{Cl}$ hydrogen-bonding interactions (Fig. S3†). These hybrid layers are further packed into a three-dimensional structure through van der Waals forces along the $c$-axis.

PEA-Mn is a canted antiferromagnet that shows weak ferromagnetism below the ordering temperature of $44.3 \mathrm{~K}\left(T_{\mathrm{C}}\right){ }^{34}$ Below $T_{\mathrm{C}}$, the isothermal magnetization along the $c$-axis (normal to the inorganic layers) reveals a spin-flop transition. As illustrated in Fig. 1b, the spin-flop is a field-induced transition where a magnetic field parallel to the easy axis of an antiferromagnet causes the spins to flop to the direction perpendicular to the field, and the critical magnetic field is called a spin-flop field $\left(H_{\mathrm{sf}}\right) .{ }^{42}$ PEA-Cu shows ferromagnetic order below $9.5 \mathrm{~K}$
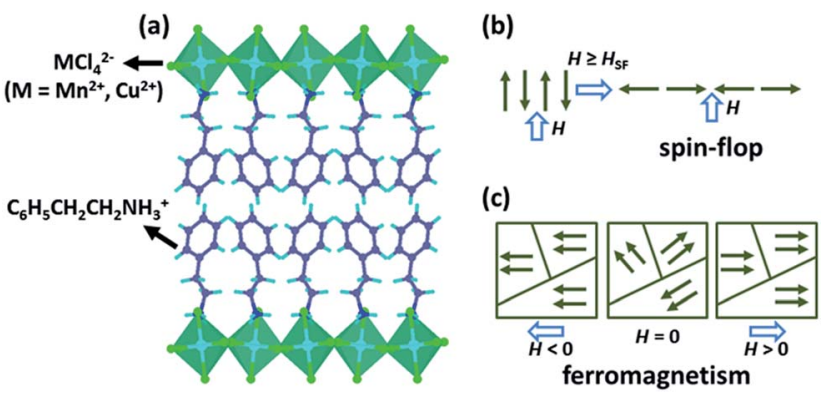

Fig. 1 Schematic diagram of the crystal structure of PEA-Mn and PEA$\mathrm{Cu}(\mathrm{a})$, the spin-flop transition in canted antiferromagnetic PEA-Mn (b), and the spin reorientation in "soft" ferromagnetic PEA-Cu (c).

(Fig. $\mathrm{S} 4 \dagger$ ) and is a "soft" ferromagnet with very small residual magnetization. Namely, as illustrated in Fig. 1c, the spin orientations of the ferromagnetic domains are randomly oriented when $H=0$, but can rapidly follow the direction of $H$ when $H>0$ or $H<0$. In addition, the field-induced spin-flop transition in PEA-Mn and the magnetization in PEA-Cu become more pronounced with decreasing temperature (Fig. S5 $\dagger$ ), indicating that the spin reorientation in both PEA-Mn and PEA-Cu are temperature dependent.

Herein, we carefully cultivated large enough and high quality single crystals for PEA-Mn and PEA-Cu, and systematically studied their magnetodielectric coupling properties by applying magnetic and electric fields on the specified face of these single crystals. We demonstrated that, for the first time, the spin reorientation in layered perovskite magnets could induce a highly reproducible magnetodielectric coupling effect, providing a promising way to design more advanced magnetodielectric materials based on layered perovskites.

\section{Experimental}

\section{Synthetic procedures}

In order to improve the sensitivity and accuracy of the results in the magnetodielectric measurements, large enough and high quality single crystals are particularly necessary. For this purpose, we increased the amounts of the raw materials: ${ }^{33,34} 50 \mathrm{mmol} 2$ phenethylamine, $25 \mathrm{mmol}$ manganese chloride dihydrate for PEA-Mn (25 mmol cupric chloride dihydrate for PEA-Cu), and $50 \mathrm{mmol}$ hydrochloric acid were dissolved in an aqueous solution and stirred for $20 \mathrm{~min}$. Then, the clear solution was kept in a quiet environment without destabilization for slow evaporation at room temperature. After about one month, a number of high quality rectangular crystals as large as several millimetres were obtained (Fig. S1 $\dagger$ ). The experimental powder X-ray patterns matched well with the simulated one, confirming the phase purity of PEA-Mn and PEA-Cu. Besides this, the X-ray patterns measured on the largest faces of the single crystals indicated that the largest face was the (001) plane normal to the $c$-axis (Fig. S2 $\dagger$ ).

\section{Physical measurements}

The silver electrodes were carefully painted onto the largest faces of the single crystals. The measurements of magnetization 
were performed on a Quantum Design physical property measurement system (PPMS) by applying a magnetic field on a single crystal along its $c$-axis. The values of the dielectric constant $\left(\varepsilon^{\prime}\right)$ at different magnetic fields and different temperatures were measured using a combination of a Tonghui TH2828A LCR meter $(E / / c)$ and PPMS $(H / / c)$ through a modified multifunctional probe with shielded cables (Quantum Design).

\section{Results and discussion}

\section{Magnetodielectric effect in PEA-Mn and PEA-Cu}

To probe the magnetodielectric coupling effect in PEA-Mn and PEA-Cu, we investigated the dielectric constant (10-1000 kHz) as a function of scanning magnetic field $(E / / c, H / / c)$ using singlecrystal samples at $3 \mathrm{~K}$. For PEA-Mn, as shown in Fig. 2a, with a scanning magnetic field $(-70 \rightarrow 0 \rightarrow 70 \mathrm{kOe})$, the dielectric constant $\left(\varepsilon^{\prime}\right)$ for all of the experimental frequencies revealed a pair of peaks accompanying a spin-flop transition at around $H_{\mathrm{sf}}( \pm 34 \mathrm{kOe})$. By defining the magnetodielectric ratio as $\mathrm{MD}=$ $\left[\varepsilon^{\prime}(H)-\varepsilon^{\prime}(0)\right] / \varepsilon^{\prime}(0)$, we can obtain a maximum MD of about $0.34 \%$ at $H_{\text {sf }}$. Among the known single-phase compounds exhibiting isothermal magnetodielectric effects, the MD of PEA$\mathrm{Mn}$ is smaller than those of the outstanding inorganic oxides such as $\mathrm{TbMnO}_{3}$ (ref. 5) and $\mathrm{DyMnO}_{3}{ }^{14}$ is comparable with those of $\mathrm{BiMnO}_{3}$ and a dysprosium-based single-molecule magnet, ${ }^{6,30}$ and is much larger than those of $\mathrm{Mn}_{3} \mathrm{O}_{4},{ }^{43} \mathrm{TbFe}_{3}$ $\left(\mathrm{BO}_{3}\right)_{4}$ (ref. 44) and the metal-formate framework $\left[\left(\mathrm{CH}_{3}\right)_{2} \mathrm{NH}_{2}\right]$ $\left[\mathrm{Fe}(\mathrm{HCOO})_{3}\right] .{ }^{25}$
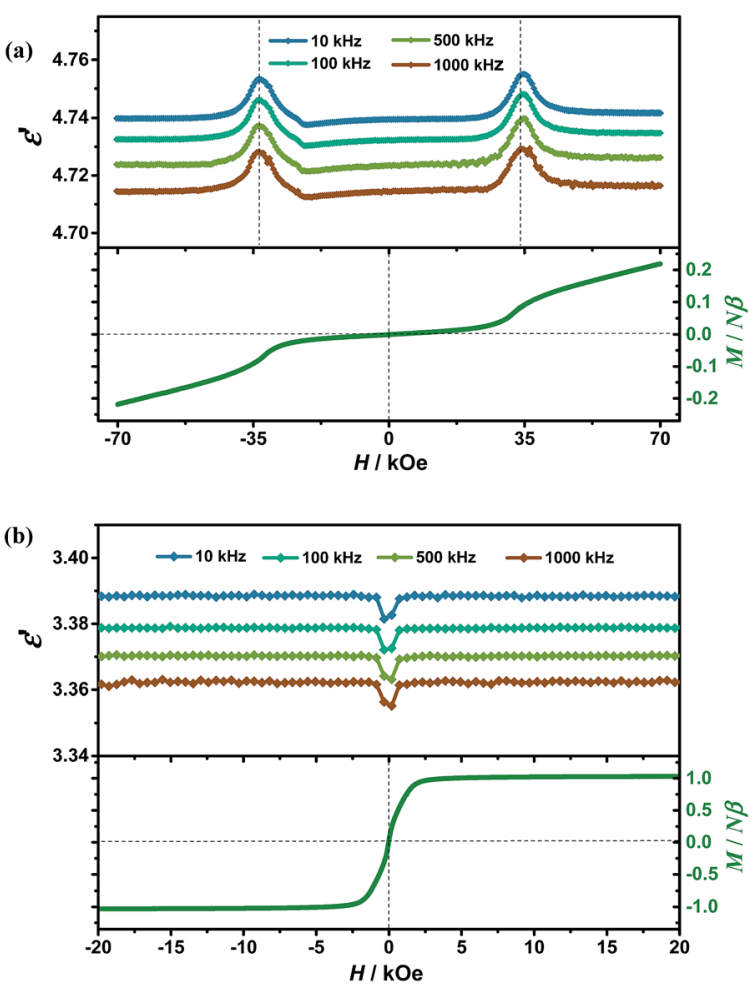

Fig. 2 Dielectric constants $\left(\varepsilon^{\prime}\right)$ and magnetization $(M)$ measured along the $c$-axis as a function of scanning magnetic field $(H)$ at $3 \mathrm{~K}$ for PEA$\mathrm{Mn}(\mathrm{a})$ and PEA-Cu (b).
For PEA-Cu, as shown in Fig. 2b, when the magnetic field is scanning from $-20 \rightarrow 0 \rightarrow 20$ kOe at $3 \mathrm{~K}, \varepsilon^{\prime}$ remains unchanged until about $-1 \mathrm{kOe}$ and then quickly decreases to a minimum value at $0 \mathrm{kOe}$. After that, it increases to the former value when $H>1 \mathrm{kOe}$. Anomalies appeared at around $0 \mathrm{kOe}$, which is the point at which the critical field of magnetization changes from negative to positive (Fig. 1c), indicating that the orientation of the spins in the "soft" ferromagnet PEA-Cu effectively alters its dielectric properties to induce a magnetodielectric coupling effect. It is interesting to find that the canted antiferromagnetic PEA-Mn only shows the maximum MD at $H_{\mathrm{sf}}( \pm 34 \mathrm{kOe})$, while the ferromagnetic PEA-Cu reaches the maximum MD $(0.21 \%)$ at a large range of about $H>1 \mathrm{kOe}$ and $H<-1 \mathrm{kOe}$. The maximum MD of PEA-Cu is comparable to that of PEA-Mn, but its applied magnetic field of about $1 \mathrm{kOe}$ is much smaller than that of PEAMn (34 kOe), demonstrating an advantage of "soft" ferromagnetism for realizing low-field controllable magnetodielectric materials.

As implicated by the coexistence of magnetic and dielectric bistabilities arising from the buckling of inorganic octahedra together with the freezing of organic cations during the thermally induced phase transitions in $\left(\mathrm{C}_{6} \mathrm{H}_{5}\left(\mathrm{CH}_{2}\right)_{4} \mathrm{NH}_{3}\right)_{2}\left[\mathrm{CuCl}_{4}\right]{ }^{41}$ a possible mechanism for the observed magnetodielectric effects in PEA-Mn and PEA-Cu could be proposed. The process of spin-reorientation in layered perovskite magnets could affect the buckling of the inorganic octahedra and then lead to a slight alternation of the polar organic cations via the hydrogenbonding interactions, and thus induce the dielectric anomalies. Notably, as no significant change in the atomic positions occurs between the magnetic phases before and after the fieldinduced spin-reorientation, the dielectric anomalies observed in PEA-Mn and PEA-Cu are field-dependent phenomena, i.e. they show a peak at the critical field, rather than the temperature-dependent phenomena commonly observed in the thermally induced phase transition (i.e. changing between two plateaus). ${ }^{41}$

\section{Magnetodielectric effect at different temperatures}

To further understand the origin of the coupled magnetic and dielectric properties, the isothermal magnetodielectric effects at different temperatures were investigated. For PEA-Mn, as shown in Fig. 3a, the magnetodielectric anomalies appear below $50 \mathrm{~K}$ and become stronger with decreasing temperature. The maximum magnetodielectric ratios at $H_{\mathrm{sf}}$ for $3,10,20,30,40$ and $50 \mathrm{~K}$ are about $0.34 \%, 0.25 \%, 0.12 \%, 0.06 \%, 0.02 \%$ and $0 \%$, respectively. As shown in the inset of Fig. 3a, the slow increase of MD with decreasing temperature is synchronous with the field-induced magnetizations during the spin-flop transitions (Fig. S5a†), further confirming the indispensable role of spin reorientation in inducing the magnetodielectric coupling effect. For PEA-Cu, the effects at different temperatures are similar to those of PEA-Mn, i.e. field-induced dielectric anomalies are observed below its magnetic ordering temperature of $9.5 \mathrm{~K}$ and become stronger with decreasing temperature (Fig. 3b). The maximum MD ratios at $3,5,7,9$ and $11 \mathrm{~K}$ are about $0.21 \%$, $0.07 \%, 0.05 \%, 0.02 \%$ and $0 \%$, respectively, and the MD shows 
(a)
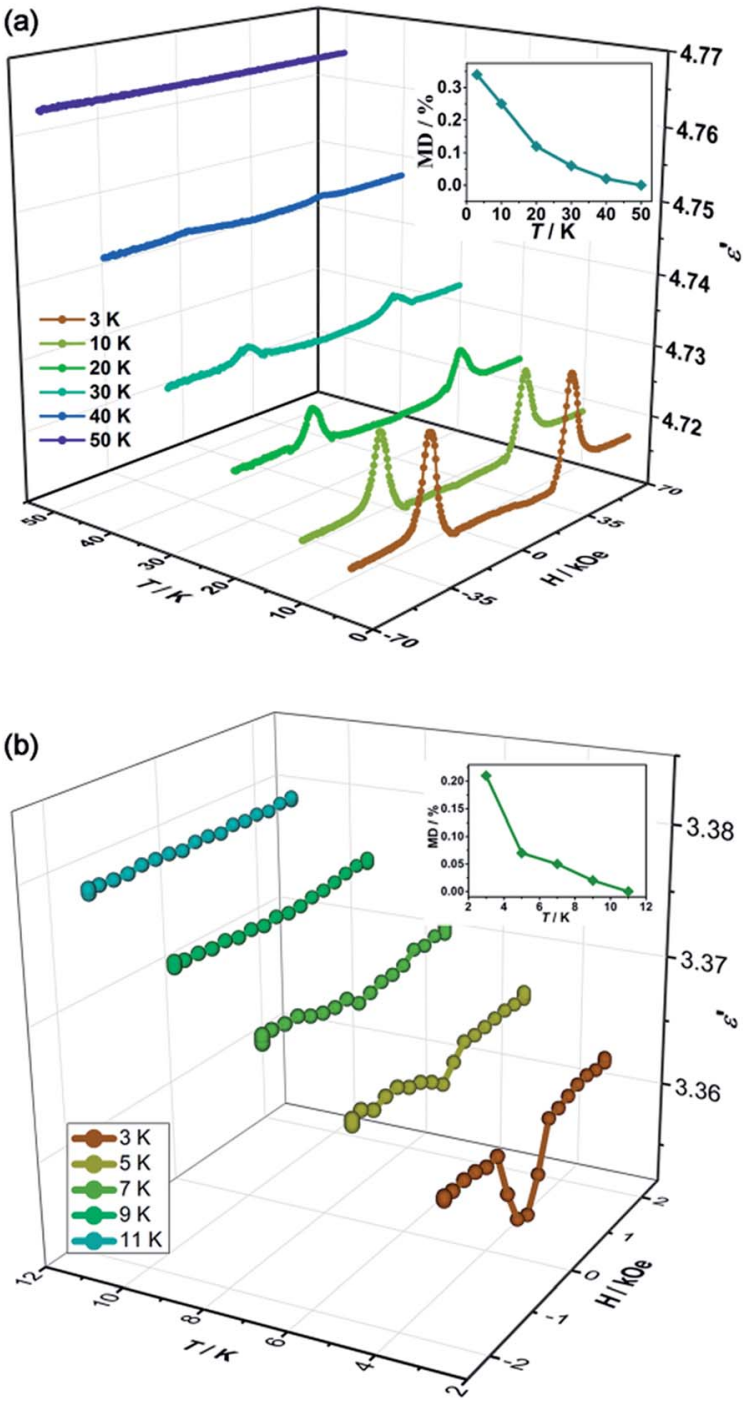

Fig. 3 Dielectric constant $\left(\varepsilon^{\prime}, 1000 \mathrm{kHz}\right)$ vs. magnetic field measured at different temperatures for PEA-Mn (a) and PEA-Cu (b). Inset: the maximum magnetodielectric ratios (MD) at different temperatures.

a temperature dependence in the multiferroic phase, i.e. being stronger at lower temperature, which is similar to the behaviour of field-induced magnetizations (Fig. S5b†). These results indicate that the magnetodielectric coupling effects in both PEA-Mn and PEA-Cu are induced by the spin reorientation. As such an effect is synchronous with the field-induced spin orientation, this may provide another way to probe/monitor the spin-reorientation via a simple dielectric measurement.

\section{Highly reproducible magnetodielectric effects}

To exclude the fake signals and confirm the intrinsic coupled dielectric and magnetic properties, it is very essential to detect the reproducibility of the magnetodielectric effect. However, studies about reproducible magnetodielectric effects are rare, and even some outstanding magnetodielectric materials such as $\mathrm{TbMnO}_{3},{ }^{5} \mathrm{DyMnO}_{3}$ (ref. 14) and $\mathrm{Ba}_{0.5} \mathrm{Sr}_{1.5} \mathrm{Zn}_{2} \mathrm{Fe}_{12} \mathrm{O}_{22}$ (ref. 45) exhibit obvious differences between the descending and ascending processes of the magnetic field. In this sense, the exploration of the fatigue phenomenon is particularly necessary and a highly reproducible magnetodielectric effect would be especially desired.

To demonstrate the reproducibility and stability of the magnetodielectric coupling effect, we set the procedure as follows: for PEA-Mn, the temperature was set at $3 \mathrm{~K}$, the magnetic fields were alternately set as 0 and $34 \mathrm{kOe}$, and the dielectric constant was measured along the $c$-axis. For comparison, the magnetization was measured using the same procedure. As shown in Fig. 4a, accompanying the alteration of the magnetic field, step-like dielectric constants $\left(\varepsilon^{\prime}\right)$ and magnetization $(M)$ are synchronously observed, which can be regarded as "on" and "off" states of dielectric and magnetic biswitches. This thus provides an extra dimension for designing multifunctional devices. Moreover, it shows almost no fatigue phenomenon along the whole experimental process, indicating that the magnetodielectric coupling effect in PEA-Mn is highly reproducible. For PEA-Cu, the experimental procedure was similar to that of PEA-Mn, but the magnetic fields were alternately set as 0 and $1 \mathrm{kOe}$. Similarly to with PEA-Mn, altering the magnetic field can switch both the dielectric constant and magnetization in PEA-Cu (Fig. 4b), confirming its highly reproducible low-field $(1 \mathrm{kOe})$ controllable magnetodielectric coupling effect. It is worth noting that, as a magnetodielectric

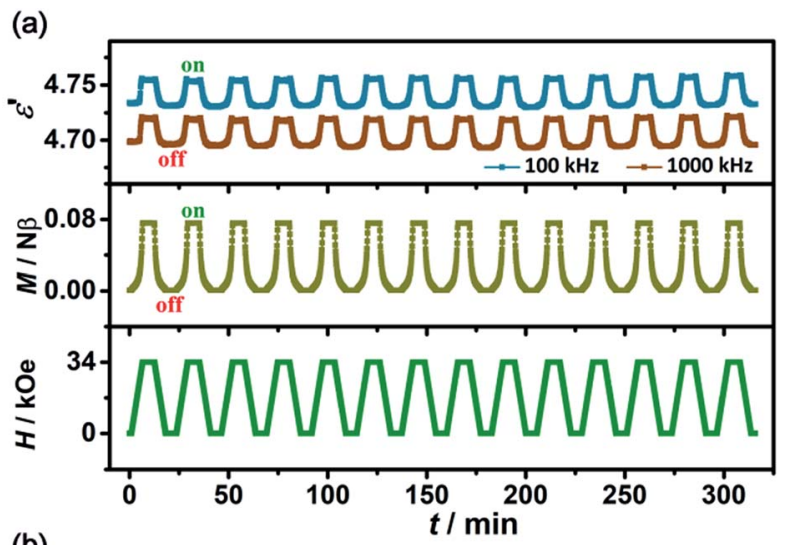

(b)

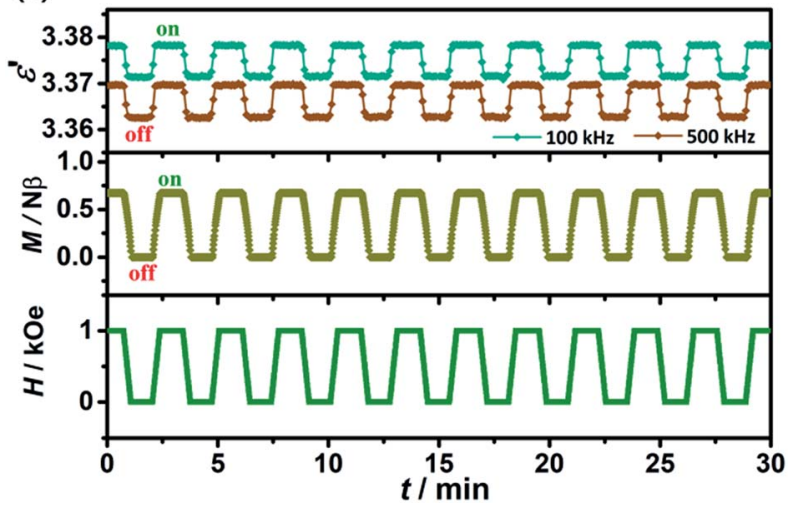

Fig. 4 Dielectric constant $\left(\varepsilon^{\prime}\right)$ and magnetization (M) for PEA-Mn (a) and PEA-Cu (b) measured at different magnetic fields $(H)$ at $3 \mathrm{~K}$. The magnetic fields for PEA-Mn were alternately set as 0 and $34 \mathrm{kOe}$, and the magnetic fields for PEA-Cu were alternately set as 0 and $1 \mathrm{kOe}$. 
coupling effect controlled by a low magnetic field is very desired but still extremely rare, ${ }^{15,22}$ the presented magnetodielectric coupling achieved via "soft" ferromagnetism in multiferroic PEA-Cu provides a new clue towards searching for advanced materials for achieving low-field magnetodielectric coupling effects.

\section{Conclusions}

In summary, the magnetodielectric coupling effects in two organic-inorganic hybrid layered perovskite compounds, i.e. PEA-Mn and PEA-Cu, were discussed. Dielectric anomalies appeared at around \pm 34 kOe for the canted antiferromagnet PEA-Mn and 0 kOe for the "soft" ferromagnet PEA-Cu. Moreover, such isothermal magnetodielectric effects in both of the layered perovskite magnets are highly reproducible, and synchronize with the field-induced magnetizations at different temperatures. Therefore, the coupled magnetic and dielectric properties in both of the layered perovskite magnets could be ascribed to the field-induced spin reorientations originating from the spin-flop transition in PEA-Mn and the magnetization reversal in PEA-Cu. The magnetodielectric coupling effects observed in PEA-Mn and PEA-Cu strongly imply that the organic-inorganic layered perovskite magnets are new, promising hybrid material systems for hosting magnetodielectric coupling effects, probably owing to their unique hydrogenbonding interactions between the inorganic magnetic layers and the organic layers. Moreover, considering the existence of the large family of organic-inorganic layered perovskite magnets, the discovery of magnetodielectric coupling effects in PEA-Mn and PEA-Cu not only opens up a promising way to design more advanced magnetodielectric materials, but also promotes the continued efforts towards the application of coupled dielectric and magnetic properties based on molecular materials.

\section{Conflicts of interest}

There are no conflicts to declare.

\section{Acknowledgements}

This work was supported by the NSFC (91422302, 21290173, 21722107, and 21671202), the BAGUI Scholar Program (2014A001), the Project of Talents Highland of Guangxi Province and the Natural Science Foundation of Guangxi Province (2014GXNSFFA118003).

\section{Notes and references}

1 M. Bibes and A. Barthelemy, Nat. Mater., 2008, 7, 425-426. 2 N. Hur, S. Park, P. A. Sharma, S. Guha and S. W. Cheong, Phys. Rev. Lett., 2004, 93, 107207.

3 M. A. Subramanian, T. He, J. Z. Chen, N. S. Rogado, T. G. Calvarese and A. W. Sleight, Adv. Mater., 2006, 18, 1737-1739.

4 G. T. Rado and V. J. Folen, Phys. Rev. Lett., 1961, 7, 310-311.
5 T. Kimura, T. Goto, H. Shintani, K. Ishizaka, T. Arima and Y. Tokura, Nature, 2003, 426, 55-58.

6 T. Kimura, S. Kawamoto, I. Yamada, M. Azuma, M. Takano and Y. Tokura, Phys. Rev. B: Condens. Matter Mater. Phys., 2003, 67, 180401.

7 G. Lawes, A. P. Ramirez, C. M. Varma and M. A. Subramanian, Phys. Rev. Lett., 2003, 91, 257208.

8 X. Wang, Y. Chai, L. Zhou, H. Cao, C. D. Cruz, J. Yang, J. Dai, Y. Yin, Z. Yuan, S. Zhang, R. Yu, M. Azuma, Y. Shimakawa, H. Zhang, S. Dong, Y. Sun, C. Jin and Y. Long, Phys. Rev. Lett., 2015, 115, 087601.

9 S. Park, Y. J. Choi, C. L. Zhang and S. W. Cheong, Phys. Rev. Lett., 2007, 98, 057601.

10 L. Zhao, T.-L. Hung, C.-C. Li, Y.-Y. Chen, M.-K. Wu, R. K. Kremer, M. G. Banks, A. Simon, M. H. Whangbo, C. Lee, J. S. Kim, I. Kim and K. H. Kim, Adv. Mater., 2012, 24, 2469-2473.

11 Y. K. Jeong, J.-H. Lee, S.-J. Ahn, S.-W. Song, H.-M. Jang, H. Choi and J. F. Scott, J. Am. Chem. Soc., 2012, 134, 14501453.

12 F. Kagawa, M. Mochizuki, Y. Onose, H. Murakawa, Y. Kaneko, N. Furukawa and Y. Tokura, Phys. Rev. Lett., 2009, 102, 057604.

13 N. Hur, S. Park, P. A. Sharma, J. S. Ahn, S. Guha and S. W. Cheong, Nature, 2004, 429, 392-395.

14 T. Goto, T. Kimura, G. Lawes, A. P. Ramirez and Y. Tokura, Phys. Rev. Lett., 2004, 92, 257201.

15 Y. Kitagawa, Y. Hiraoka, T. Honda, T. Ishikura, H. Nakamura and T. Kimura, Nat. Mater., 2010, 9, 797-802.

16 J.-P. Zhao, J. Xu, S.-D. Han, Q.-L. Wang and X.-H. Bu, Adv. Mater., 2017, 29, 1606966.

17 F. Kagawa, S. Horiuchi, M. Tokunaga, J. Fujioka and Y. Tokura, Nat. Phys., 2010, 6, 169-172.

18 H. Cui, Z. Wang, K. Takahashi, Y. Okano, H. Kobayashi and A. Kobayashi, J. Am. Chem. Soc., 2006, 128, 15074-15075.

19 P. Jain, V. Ramachandran, R. J. Clark, H. D. Zhou, B. H. Toby, N. S. Dalal, H. W. Kroto and A. K. Cheetham, J. Am. Chem. Soc., 2009, 131, 13625-13627.

20 G.-C. Xu, W. Zhang, X.-M. Ma, Y.-H. Chen, L. Zhang, H.-L. Cai, Z.-M. Wang, R.-G. Xiong and S. Gao, J. Am. Chem. Soc., 2011, 133, 14948-14951.

21 D.-W. Fu, W. Zhang, H.-L. Cai, Y. Zhang, J.-Z. Ge, R.-G. Xiong, S. D. Huang and T. Nakamura, Angew. Chem., Int. Ed., 2011, 50, 11947-11951.

22 L.-H. Chen, J.-B. Guo, X. Wang, X.-W. Dong, H.-X. Zhao, L.-S. Long and L.-S. Zheng, Adv. Mater., 2017, 29, 1702512.

23 W. Wang, L.-Q. Yan, J.-Z. Cong, Y.-L. Zhao, F. Wang, S.-P. Shen, T. Zou, D. Zhang, S.-G. Wang, X.-F. Han and Y. Sun, Sci. Rep., 2013, 3, 2024.

24 Y. Tian, A. Stroppa, Y. Chai, L. Yan, S. Wang, P. Barone, S. Picozzi and Y. Sun, Sci. Rep., 2014, 4, 6062.

25 Y. Tian, S. Shen, J. Cong, L. Yan, S. Wang and Y. Sun, J. Am. Chem. Soc., 2016, 138, 782-785.

26 L. C. Gomez-Aguirre, B. Pato-Doldan, J. Mira, S. CastroGarcia, M. A. Senaris-Rodriguez, M. Sanchez-Andujar, J. Singleton and V. S. Zapf, J. Am. Chem. Soc., 2016, 138, 1122-1125. 
27 J.-B. Guo, L.-H. Chen, D. Li, H.-X. Zhao, X.-W. Dong, L.-S. Long, R.-B. Huang and L.-S. Zheng, Appl. Phys. Lett., 2017, 110, 192902.

28 H.-L. Cai, Y. Zhang, D.-W. Fu, W. Zhang, T. Liu, H. Yoshikawa, K. Awaga and R.-G. Xiong, J. Am. Chem. Soc., 2012, 134, 18487-18490.

29 M. Ackermann, D. Bruning, T. Lorenz, P. Becker and L. Bohaty, New J. Phys., 2013, 15, 123001.

30 Y.-X. Wang, Y. Ma, Y. Chai, W. Shi, Y. Sun and P. Cheng, J. Am. Chem. Soc., 2018, 140, 7795-7798.

31 M. Ackermann, T. Lorenz, P. Becker and L. Bohaty, J. Phys.: Condens. Matter, 2014, 26, 506002.

32 L. F. Larkworthy and A. Yavari, J. Chem. Soc., Dalton Trans., 1978, 1236-1240.

33 A. O. Polyakov, A. H. Arkenbout, J. Baas, G. R. Blake, A. Meetsma, A. Caretta, P. H. M. van Loosdrecht and T. T. M. Palstra, Chem. Mater., 2011, 24, 133-139.

34 S. H. Park, I. H. Oh, S. Park, Y. Park, J. H. Kim and Y. D. Huh, Dalton Trans., 2012, 41, 1237-1242.

35 P. Day, Acc. Chem. Res., 1978, 12, 236-243.

36 L. J. de Jongh, J. Appl. Phys., 1969, 40, 1363.

37 J. Han, S. Nishihara, K. Inoue and M. Kurmoo, Inorg. Chem., 2015, 54, 2866-2874.
38 J. Han, S. Nishihara, K. Inoue and M. Kurmoo, Inorg. Chem., 2014, 53, 2068-2075.

39 J. C. Crowley, H. W. Dodgen and R. D. Willett, J. Phys. Chem., 1982, 86, 4046-4055.

40 B. Kundys, A. Lappas, M. Viret, V. Kapustianyk, V. Rudyk, S. Semak, C. Simon and I. Bakaimi, Phys. Rev. B: Condens. Matter Mater. Phys., 2010, 81, 224434.

41 B. Huang, B.-Y. Wang, Z.-Y. Du, W. Xue, W.-J. Xu, Y.-J. Su, W.-X. Zhang, M.-H. Zeng and X.-M. Chen, J. Mater. Chem. C, 2016, 4, 8704-8710.

42 X.-Y. Wang, L. Wang, Z.-M. Wang, G. Su and S. Gao, Chem. Mater., 2005, 17, 6369-6380.

43 R. Tackett, G. Lawes, B. C. Melot, M. Grossman, E. S. Toberer and R. Seshadri, Phys. Rev. B: Condens. Matter Mater. Phys., 2007, 76, 024409.

44 U. Adem, L. Wang, D. Fausti, W. Schottenhamel, P. H. M. van Loosdrecht, A. Vasiliev, L. N. Bezmaternykh, B. Büchner, C. Hess and R. Klingeler, Phys. Rev. B: Condens. Matter Mater. Phys., 2010, 82, 064406.

45 T. Kimura, G. Lawes and A. P. Ramirez, Phys. Rev. Lett., 2005, 94, 137201. 